\title{
Association between disease activity and ischaemia- modified albumin in patients with ulcerative colitis
}

\author{
Serkan Ipek ${ }^{1}$, Hulya Yalcin ${ }^{2}$, Burak Toprak ${ }^{2}$ \\ ${ }^{1}$ Department of Gastroenterology, Bakirkoy Dr. Sadi Konuk Training and Research Hospital, Istanbul, Turkey \\ ${ }^{2}$ Department of Biochemistry, Tepecik Training and Research Hospital, Izmir, Turkey
}

Gastroenterology Rev 2022; 17 (3): 203-206

DOI: https://doi.org/10.5114/pg.2021.109664

Key words: ulcerative colitis, ischaemia-modified albumin.

Address for correspondence: Serkan Ipek, Department of Gastroenterology, Bakirkoy Dr. Sadi Konuk Training and Research Hospital, Istanbul, Turkey, e-mail: serkanipek@hotmail.com

\begin{abstract}
Introduction: An increased level of ischaemia-modified albumin (IMA) is not specific for cardiac ischaemia and has been shown to be elevated in many other conditions causing oxidative stress.

Aim: To assess the association between IMA and the disease activity in ulcerative colitis (UC), in which oxidative stress is thought to play a role in its pathogenesis.

Material and methods: A total of 57 patients with ulcerative colitis (30 with active disease and 27 in remission) and 29 healthy controls were included in the study. IMA levels in those with active disease, those in remission, and healthy controls were compared. The correlations between IMA and other acute phase reactants were also assessed in the patient group.

Results: Significantly higher levels of IMA were found in patients with active UC as compared to those in remission and controls $(p<0.001)$. Patients in remission and control subjects did not differ significantly in terms of IMA levels. Also, IMA correlated with C-reactive protein and erythrocyte sedimentation rate, while it did not correlate with white blood cell count and platelet count.

Conclusions: Our results suggest that IMA, a marker of oxidative stress, may be a useful parameter for assessing the disease activity in patients with ulcerative colitis.
\end{abstract}

\section{Introduction}

The $\mathrm{N}$ terminal of albumin molecule is the primary site where transitional metals such as cobalt, copper, and nickel bind [1]. During acute ischaemic conditions, the metal binding capacity of albumin for transition metals is reduced to what is commonly known as ischaemia-modified albumin (IMA) [2]. The precise mechanism of how IMA is produced is not known but appears to be related to the production of reactive oxygen species that modify the metal binding sites $[3,4]$ High serum IMA level has been proposed as a sensitive marker for the diagnosis of myocardial ischaemia before the onset of irreversible cardiac injury in patients presenting with typical acute chest pain. Serum IMA was measured using the albumin cobalt binding (ACB) test, which measures the binding capacity of albumin to cobalt [1]. However, IMA levels are also known to increase in other ischaemic conditions and in diseases such as advanced cancer, systemic sclerosis, intrauterine disorders, and end-stage renal disease due to free radical production $[5,6]$. Thus, IMA has been identified as a marker of oxidative stress in many previous studies [7-9].

Ulcerative colitis (UC) is a chronic condition characterized by recurrent episodes of colon inflammation, which is the underlying cause of many of the symptoms and findings of UC. Therefore, the diagnosis and follow-up of the inflammation are critically important for the clinical management of the disease [10]. A principle mechanism responsible for tissue injury in UC is the oxidative stress due to excessive release of reactive oxygen metabolites (ROM) [11].

In the past decade, several laboratory markers have been evaluated for their efficacy in determining disease activity, although none has proven ideal [12]. This has led to a search for novel markers that more effectively reflect the disease activity. 


\section{Aim}

In this study, the association between disease activity in UC, in which oxidative stress is thought to play a role in the pathogenesis, and IMA, a marker of oxidative stress, has been evaluated.

\section{Material and methods}

This study was conducted at the Department of Gastroenterology, Izmir Katip Celebi University, Ataturk Research and Training Hospital. A total of 57 patients with ulcerative colitis (30 with active disease and 27 in remission) and 29 healthy controls were included in the study. The endoscopic disease activity was estimated using the Rachmilewitz activity index. Patients with an activity index lower than 4 were considered to be in remission, while patients with an activity index higher than 4 were identified as actively diseased. Albumin, IMA, C-reactive protein (CRP), erythrocyte sedimentation rate (ESR), white blood cell count (WBC), and platelet count (PLT) were examined in UC patients, while IMA and albumin were determined among controls. The IMA levels between those with active UC, UC in remission, and healthy controls were compared. Also, the correlation between IMA and other parameters was examined in the patient group.

All laboratory measurements were performed on fasting blood samples. IMA level (reduced cobalt to albumin binding capacity) was analysed according to the method defined by Bar-Or et al. [1]. Two hundred microlitres $(\mu \mathrm{l})$ of patient serum was placed into glass tubes and $50 \mu \mathrm{l}$ of $0.1 \% \mathrm{CoC}_{12}+6 \mathrm{H}_{2} \mathrm{O}$ (Sigma-Aldrich) was added. After gentle shaking, the mixture was left undisturbed for $10 \mathrm{~min}$ to ensure sufficient cobalt albumin binding. Then, $50 \mu \mathrm{l}$ of $1.5 \mathrm{mg} / \mathrm{ml}$ dithiothreitol (DTT) (Sigma-Aldrich) was added as a colouring agent. After $2 \mathrm{~min} 1 \mathrm{ml}$ of $0.9 \% \mathrm{NaCl}$ was added to quench the reaction. A control sample was prepared for every sample. At the DTT addition stage, $50 \mu \mathrm{l}$ of distilled water was used instead of $50 \mu$ of $1.5 \mathrm{mg} / \mathrm{ml}$ DTT to obtain a control sample without DTT. Sample absorbencies were analysed at $470 \mathrm{~nm}$ using spectrophotometry (Shimadzu UVmini1240; Shimadzu, Tokyo, Japan). A sample without DTT was used as a blank. The results were reported as absorbance units (ABSU). Serum albumin leve was measured with an Olympus AU 5800 autoanalyzer (Olympus, Tokyo, Japan). CRP was measured using an Abbott (Lot No: 81067HW00) kit with an Abbott C16000 device immunoturbidimetrically. For WBC and PLT analyses, blood samples were placed in complete blood count tubes containing $2 \mathrm{ml}$ of EDTA, and the measurements were performed on a Coulter complete blood count device. ESR was determined with a Vacuplus ESR-120 fully automatic device, and the sedimentation rate at $1 \mathrm{~h}$ was expressed as $\mathrm{mm} / \mathrm{h}$. The study protocol was approved by the local hospital ethics committee.

\section{Statistical analysis}

Statistical analysis were performed using SPSS software (version 17, SPSS Inc., Chicago, IL). The conformity of continuous variables to Gauss distribution was tested using Kolmogorov-Smirnov analysis. For the comparison of CRP, WBC, ESR, and PLT between patients with active disease and patients in remission, the Mann-Whitney $U$ test was used, because these did not exhibit a normal distribution. Subgroup comparisons with respect to IMA were based on one-way ANOVA analysis, and pairwise group comparisons were performed using Tukey's test due to equality in variance. Spearman correlation analysis was used to assess the correlation between IMA and CRP, ESR, WBC, and PLT. A performance assessment for IMA and other parameters was performed using receiver operator characteristics (ROC) analysis.

\section{Results}

The mean age in the whole group of 86 patients ( 51 male, 35 female) was $45.2 \pm 13.4$ years. Of the patients with active UC, 21 (70\%) were male and 9 (30\%) were female, with a mean age of $45.2 \pm 14.6$ years. Of the patients in remission, 13 (48.1\%) and 14 (51.9\%) were male and female, respectively, with a mean age of 48.4 \pm 14.3 years. The healthy control group comprised 17 (58.6\%) male and 12 (41.4\%) female individuals, with a mean age of $42.3 \pm 10.5$ years.

Significantly higher levels of IMA were detected among UC patients with active disease as compared to those in remission and controls $(p<0.001)$. Also, albumin levels were significantly lower among those with active disease than in other study participants. No significant differences between controls and patients in remission were observed with respect to IMA ( $p=0.908)$. In terms of CRP, ESR, WBC, and PLT measurements, patients with active disease had significantly higher CRP and ESR than those in remission ( $p<0.001$ ), while the 2 groups did not differ significantly in WBC and PLT (Table I). The analyses in the patient group examining the correlation between IMA and other parameters showed a correlation between IMA and CRP and ESR, while no such correlations could be found with WBC and PLT (Table II). IMA emerged as the single most important marker of disease activity in UC, as shown by ROC curve analysis (Figure 1) (area under curve (AUC) for IMA was 0.85), followed by CRP, ESR, WBC, and PLT counts (Table III).

\section{Discussion}

Although UC is a disease entity of unknown aetiology, dysfunctional immune regulation of the colon is be- 
Table I. Comparison of IMA, CRP, ESR, WBC, and PLT levels between the study groups

\begin{tabular}{|c|c|c|c|c|}
\hline \multirow[t]{2}{*}{ Parameter } & \multicolumn{2}{|c|}{ UC group } & \multirow{2}{*}{$\begin{array}{l}\text { Control group } \\
\quad(n=29)\end{array}$} & \multirow[t]{2}{*}{$P$-value } \\
\hline & Active $(n=30)$ & Remission $(n=27)$ & & \\
\hline Albumin $[\mathrm{g} / \mathrm{dl}]^{*}$ & $3.9 \pm 0.5$ & $4.4 \pm 0.3$ & $4.4 \pm 0.4$ & $<0.001^{\mathrm{a}}$ \\
\hline IMA $[\mathrm{ABSU}]^{*}$ & $0.51 \pm 0.13$ & $0.30 \pm 0.13$ & $0.29 \pm 0.09$ & $<0.001^{a}$ \\
\hline $\mathrm{CRP}[\mathrm{mg} / \mathrm{dll}]^{\star \star}$ & $1.95(0.10-21.9)$ & $0.3(0.10-3.90)$ & & $<0.001^{b}$ \\
\hline $\mathrm{ESR}[\mathrm{mm} / \mathrm{h}]^{\star \star}$ & $35(3-103)$ & $10(2-55)$ & & $<0.001^{b}$ \\
\hline$W B C[K / \mu l]^{* *}$ & $8220(4980-17800)$ & 7160 (2940-11000) & & $0.027^{b}$ \\
\hline PLT $[K / \mu l]^{\star \star}$ & 344 (178-751) & 284 (169-489) & & $0.014^{b}$ \\
\hline
\end{tabular}

*Values are expressed as mean $\pm S D .{ }^{* *}$ Values are expressed as median (min.-max.). ${ }^{a}$ One-way ANOVA test, $p$-value. ${ }^{b}$ Mann-Whitney U test $p$-value.

Table II. Correlation of IMA with other inflammatory parameters (Pearson correlation)

\begin{tabular}{lc} 
Parameter & IMA \\
\hline CRP & $r=0.522, p \leq 0.001$ \\
\hline ESR & $r=0.395, p \leq 0.001$ \\
\hline WBC & $r=0.127, p=0.348$ \\
\hline PLT & $r=0.146, p=0.277$
\end{tabular}

lieved to play a major role in its development [13]. Of the factors involved in immune regulation, abnormally elevated levels of reactive oxygen species (ROS) are noted in patients with UC. The destructive effects of ROS may contribute to the onset and progression of UC [14-17].

Oxidative stress results from an imbalance between ROS production and antioxidant defences [9] that is caused either by the failure of the antioxidant system activity or by excessive ROS production. Both parameters are thought to play a role in a variety of autoimmune conditions (e.g. systemic lupus erythematous, pemphigus foliaceus, diabetes mellitus, primary Sjogren's syndrome, and inflammatory bowel disease) [18-20]. One of the basic mechanisms of tissue injury in $U C$ is represented by the oxidative stress resulting from excessive release of reactive oxygen metabolites (ROM) [11]. Previous studies found significantly increased ROM production in colonic biopsy samples from UC patients as compared to normal mucosal samples, and further discovered that ROM significantly correlated with the disease activity $[21,22]$.

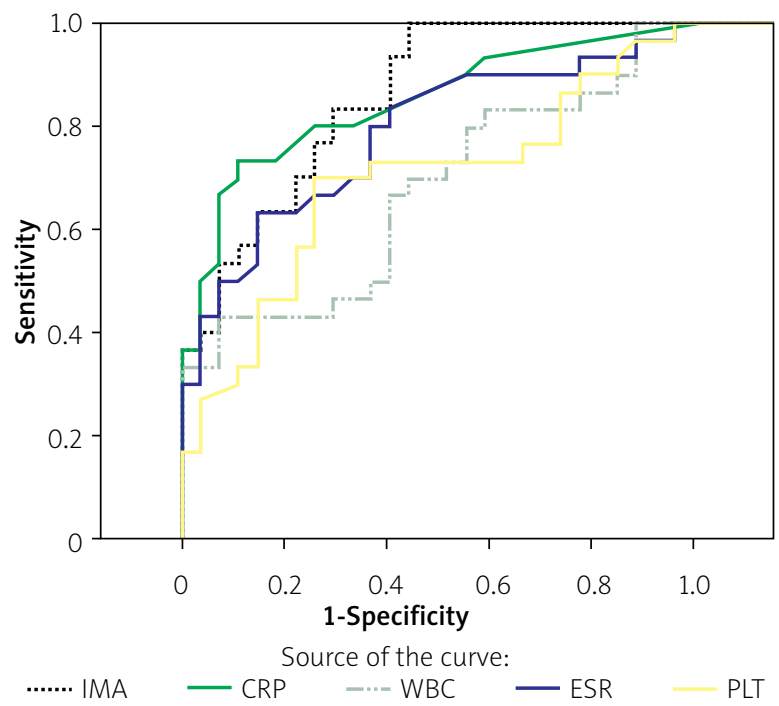

Figure 1. ROC curve analyses of laboratory parameters

IMA is not specific to cardiac ischaemia and may also be elevated in individuals exposed to oxidative stress due to other conditions. IMA has been proposed to represent a biomarker for both ischaemia-related conditions and other disorders involving oxidative stress [23]. Also, IMA may be elevated in many other conditions including liver cirrhosis, advanced cancer, acute infections, brain ischaemia, end-stage renal disease, and intrauterine ischaemia [24]. However, its current use is restricted to the assessment of ischaemia, with FDA approval granted exclusively for the diagnosis

Table III. Diagnostic accuracy and best cut-off values for the prediction of ulcerative colitis active disease

\begin{tabular}{lccccc} 
Parameter & AUC & $\mathbf{9 5 \% ~ C l}$ & Best cut-off & Sensitivity (\%) & Specificity (\%) \\
\hline IMA $[\mathrm{ABSU}]$ & 0.853 & $0.757-0.949$ & 0.379 & 83 & 76 \\
\hline CRP $[\mathrm{mg} / \mathrm{dll}]$ & 0.845 & $0.743-0.947$ & 0.95 & 73 & 88 \\
\hline ESR $[\mathrm{mm} / \mathrm{h}]$ & 0.782 & $0.662-0.902$ & 24.5 & 63 & 85 \\
\hline WBC $[\mathrm{K} / \mu \mathrm{l}]$ & 0.670 & $0.530-0.811$ & 9670 & 43 & 92 \\
\hline PLT $[\mathrm{K} / \mu \mathrm{ll}]$ & 0.690 & $0.550-0.829$ & 314 & 70 & 74
\end{tabular}


of cardiac ischaemia [8]. Elevated IMA levels in patients with non-ischaemic conditions suggest the presence of oxidative stress and systemic inflammation of non-cardiac origin $[7,25,26]$.

In this study, the association between IMA levels and UC activity was examined based on the important role of oxidative stress in the pathogenesis of UC. Thus, a significantly higher IMA was found in those with active UC as compared to those in remission or controls ( $p<0.001)$. On the other hand, patients in remission and controls did not differ significantly with respect to IMA. Also, IMA correlated with other markers of disease activity, i.e. CRP and ESR, while no such correlations were found for WBC and PLT. The ROC analysis of our study suggested that among the parameters tested, IMA represented the single most important marker $(A \cup C=0.85)$ for $U C$ activity.

\section{Conclusions}

The observation that significantly higher levels of IMA were found among active UC patients versus those in remission and controls suggests that this parameter may be useful in the assessment of disease activity in patients with UC. Further studies are warranted to better define the role of IMA in UC activation.

\section{Conflict of interest}

The authors declare no conflict of interest.

\section{References}

1. Bar-Or D, Lau E, Winkler JV. A novel assay for cobalt-albumin binding and its potential as a marker for myocardial ischemia - a preliminary report. J Emerg Med 2000; 19: 311-5.

2. Lippi G, Montagnana M, Guidi GC. Albumin cobalt binding and ischemia modified albumin generation: an endogenous response to ischemia? Int J Cardiol 2006; 108: 410-1.

3. Bar-Or D, Curtis G, Rao N, et al. Characterization of the $\mathrm{Co}(2+)$ and $\mathrm{Ni}(2+)$ binding amino-acid residues of the $\mathrm{N}$-terminus of human albumin. Eur J Biochem 2001; 268: 42-7.

4. Gidenne S, Ceppa F, Fontan E, et al. Analytical performance of the Albumin Cobalt Binding (ACB) test on the Cobas MIRA Plus analyzer. Clin Chem Lab Med 2004; 42: 455-61.

5. Sbarouni E, Georgiadou P, Voudris V. Ischemia modified albümin changes: review and clinical implications. Clin Chem Lab Med 2011; 49: 177-84.

6. Turedi S, Cinar O, Yavuz I, et al. Differences in ischemia-modified albumin levels between end stage renal disease patients and the normal population. J Nephrol 2010; 23: 335-40.

7. Yang LX, Ma SG, Liu H, Xu W. Influence of obstructive sleep apnea on serum butyrylcholinesterase activity and ischemia-modified albumin levels. Clinics 2013; 68: 968-73.

8. Gaze DC. Ischemia modified albumin: a novel biomarker for the detection of cardiac ischemia. Drug Metab Pharmacokinet 2009; 24: 333-41.
9. Bouzid D, Gargouri B, Mansour RB, et al. Oxidative stress markers in intestinal mucosa of Tunisian inflammatory bowel disease patients. Saudi J Gastroenterol 2013; 19: 131-5.

10. Konikoff MR, Denson LA. Role of fecal calprotectin as a biomarker of intestinal inflammation in inflammatory bowel disease. Inflamm Bowel Dis 2006; 12: 524-34.

11. Kruidenier L, Verspaget HW. Review article: oxidative stress as a pathogenic factor in inflammatory bowel disease: radicals or ridiculous? Aliment Pharmacol Ther 2002; 16: 1997-2015.

12. Vermeire $S$, Van Assche $G$, Rutgeerts P. Laboratory markers in IBD: useful, magic, or unnecessary toys? Gut 2006; 55: 426-31.

13. Shih DQ, Targan SR, McGovern D. Recent advances in IBD pathogenesis: genetics and immunobiology. Curr Gastroenterol Rep 2008; 10: 568-75.

14. Pavlick KP, Laroux FS, Fuseler J, et al. Role of reactive metabolites of oxygen and nitrogen in inflammatory bowel disease. Free Radic Biol Med 2002; 33: 311-22.

15. Pravda J. Radical induction theory of ulcerative colitis. World J Gastroenterol 2005; 11: 2371-84.

16. Karp SM, Koch TR. Oxidative stress and antioxidants in inflammatory bowel disease. Dis Mon 2006; 52: 199-207.

17. Rezaie A, Parker RD, Abdollahi M. Oxidative stress and pathogenesis of inflammatory bowel disease: an epiphenomenon or the cause? Dig Dis Sci 2007; 52: 2015-21.

18. Kurien BT, Scofield RH. Free radical mediated peroxidative damage in systemic lupus erythematosus. Life Sci 2003; 73: 1655-66.

19. de M Bandeira S, da Fonseca LJ, da S Guedes G, et al. Oxidative stress as an underlying contributor in the development of chronic complications in diabetes mellitus. Int J Mol Sci 2013; 14: 3265-84.

20. Sklyarov AY, Panasyuk NB, Fomenko IS. Role of nitric oxide-synthase and cyclooxygenase/lipooxygenase systems in development of experimental ulcerative colitis. J Physiol Pharmacol 2011; 62: 65-73.

21. Sedghi S, Fields JZ, Klamut M, et al. Increased production of luminol enhanced chemiluminescence by the inflamed colonic mucosa in patients with ulcerative colitis. Gut 1993; 34: 1191-7.

22. Lih-Brody L, Powell SR, Collier KP, et al. Increased oxidative stress and decreased antioxidant defenses in mucosa of inflammatory bowel disease. Dig Dis Sci 1996; 41: 2078-86.

23. Apple FS, Wu AH, Mair J, et al.; Committee on Standardization of Markers of Cardiac Damage of the IFCC. Future biomarkers for detection of ischemia and risk stratification in acute coronary syndrome. Clin Chem 2005; 51: 810-24.

24. Ma SG, Jin Y, Hu W, et al. Evaluation of ischemia-modified albumin and C-reactive protein in type 2 diabetics with and without ketosis. Biomark Insights 2012; 7: 19-26.

25. Chen CY, Tsai WL, Lin PJ, Shiesh SC. The value of serum ischemia-modified albumin for assessing liver function in patients with chronic liver disease. Clin Chem Lab Med 2011; 49: 1817-21.

26. Ma SG, Wei CL, Hong B, Yu WN. Ischemia-modified albumin in type 2 diabetic patients with and without peripheral arterial disease. Clinics 2011; 66: 1677-80.

Received: 27.06.2021

Accepted: 27.07.2021 